\title{
Phonon Density of States and Debye Temperatures of BCC Transition Metals
}

\author{
A. R. Jani \\ Department of Physics, Sardar Patel University, Vallabh Vidyanagar 388 120, Gujarat, India
}

Z. Naturforsch. 40a, 834-842 (1985); received May 20, 1985

A screened shell phenomenological model is applied to determine the density of phonon states and Debye temperatures of six bcc transition metals. The theoretical results are discussed in the light of existing experimental and other theoretical data. It has been found that the results obtained in the present study are better than those reported previously on the basis of phenomenological models. Moreover, it should be emphasized that in the present work a fine mesh of 64000 evenly spaced vector has been employed to generate the phonon frequency spectra.

\section{Introduction}

Transition metals and their compounds provide one of the most fascinating areas in the study of phonons in crystal lattices. This is because, in addition to the richness and variety of structure of their phonon dispersion curves, these materials often exhibit the phenomena of lattice instabilities and relatively high temperature $(8 \mathrm{~K}-23 \mathrm{~K})$ superconductivity, in which phonons play a vital role. Until the last two decades, a considerable interest has been shown $[1-14]$ in the study of lattice vibrations in bec transition metals by measuring the phonon frequencies from inelastic neutron scattering. The observation of the fine structure characteristics in the phonon dispersion curves of these metals has also stimulated much interest in the allied properties. It is evident that the physical properties of the crystal which depend on the motion of the constituent particles require for their detailed explanation a knowledge of the density of phonon states. In the calculation of such a physical property, care should be taken while averaging the phonon frequencies over the whole spectrum. Therefore it is necessary to have a proper lattice dynamical model capable of generating a proper frequency distribution. Ultimately, a comparative study of results based on such calculations with accurate experimental determinations provides a test of the phonon characteristics, and thus of the model used.

Reprint requests to Dr. A. R. Jani, Department of Physics, Sardar Patel University, Vallabh Vidyanagar 388120. Gujarat, India.
Recently, we have put forth a screened shell phenomenological model for the lattice dynamics of cubic metals [15]. It has also been modified for bcc transition metals to compute the phonon dispersion relations in the symmetry directions [16]. As these models have explained the dispersion curves of alkali, noble and transition metals quite satisfactorily, it was thought worthwhile to examine its applicability to other properties also. In the present paper, the phonon density of states and the temperature variation of the Debye temperature $\theta_{\mathrm{D}}$ (obtained from the constant volume lattice specific heats) of tungsten, molybdenum, alpha-iron, chromium, tantalum and vanadium have been studied with the help of screened shell phenomenological models $[15,16]$.

\section{Theory and Numerical Computation}

The derivation of the dynamical matrix, its diagonalization and hence the eigenfrequency $(v)$ versus eigenvector $(q)$ relations in the symmetry directions, the elastic constants and other pertinent data are now well established and will not be given here. In fact, we have striven to avoid duplication of the material discussed in our previous papers [15-18].

To estimate the value of the Debye temperature, it is essential to determine the frequency spectrum of the lattice vibrations at a suitable number of points in an irreducible section of the first Brillouin zone. This frequency distribution has been obtained by the numerical sampling of frequencies according 
to Blackmann's technique [19-21] over a discret subdivision in the wave vector space.

In order to have a large survey of frequencies, in the present work we have considered a mesh of 64000 evenly spaced wave vectors in the first Brillouin zone. For this purpose, the first Brillouin zone was divided into $40 \times 40 \times 40$ miniature cells with axes $1 / 40$ of the length of the reciprocal lattice cell. Consideration of Born's cyclic boundary condition and the symmetry property of the lattice reduces the 64000 points to 1661 non-equivalent points lying within an irreducible 1/48 part of the first Brillouin zone. Each point was weighted according to the number of points equivalent to it by symmetry. Care was taken in giving the proper weights to points lying on the surface, edges and corners of the Brillouin zone. The 192000 phonon eigenfrequencies corresponding to 64000 eigenvectors in the zone were computed from the solution of the secular determinant formed by the dynamical matrix of our previously proposed theory [16].

To evaluate the frequency distribution function $g(v)$, counts of the number of frequencies falling into intervals of a $1 / 100$ part of the maximum frequency were used to construct a frequency histogram. It should be noted that in most of the previous calculations [22-32] a much coarser mesh was employed.

Using the information about the phonon density of states evaluated in the above way, the lattice specific heat at constant volume $\left(C_{\mathrm{v}}\right)$ was calculated in the usual way. Corresponding values of the Debye temperature $\left(\theta_{\mathrm{D}}\right)$ at different temperatures were determined by employing standard tables [33] of $C_{\mathrm{v}} / 3 R$ versus $\theta_{\mathrm{D}} / T$.

\section{Results and Discussion}

Tungsten: Chen and Brockhouse [32] have calculated the frequency distribution function $g(v)$ using 23 force constants obtained from the 8 th neighbour best fit curves for the experimental phonon frequencies. They have reported two pronounced peaks at the frequencies 4.6 and 6.3 (in THz). Comparing present results (Fig. 1) it is evident that the positions of the respective peaks are in close agreement with their results. The computed result (Fig. 1) is also found to be better than the previous results $[24,28]$.
Clusius and Franzosini [34] have experimentally determined the specific heats in the case of tungsten. The earlier measurements $[34,35]$ for this metal were for the temperature ranges up to and above $91 \mathrm{~K}$, respectively. The position regarding the coefficients of the electronic specific heat $(\gamma)$ is not clear enough for tungsten, and there is a considerable divergence in the values reported by different workers [34, 37-42]. The experimental values, however, are $1.8 \pm 0.7$ [37], $3.5 \pm 0.2$ [38], 2.89 [39] and $2.65 \pm 0.24$ [40], all in $10^{-4} \mathrm{cal} / \mathrm{mol} \mathrm{deg}^{2}$. Manning and Chodorow [42] have computed $\gamma$ to be $4.8 \times 10^{-4}$ $\mathrm{cal} / \mathrm{mol} \mathrm{deg}^{2}$. Clusius and Franzosini [34] have recommended the values 2.7 and $4.9 \times 10^{-4} \mathrm{cal} / \mathrm{mol}$ $\cdot \mathrm{deg}^{2}$ for temperatures below and above $20 \mathrm{~K}$, respectively. We have evaluated lattice specific heats from the data of Clusius and Franzosini [34]. In the $\theta_{\mathrm{D}}$ vs. $T$ plot for this metal (Fig. 2) the values of $\theta_{\mathrm{D}}$ of other authors, referred above, have also been shown. It can be seen therefrom that our theoretical values agree excellently with the experimental values.

Molybdenum: Walker and Egelstaff [43] and Powell et al. [44] have determined the frequency spectrum of molybdenum employing a Born-Von Karman model fitted with inelastic neutron scattering data. We have selected the recent data [44] for the comparison in which the frequency spectrum has been generated by fitting a 7 th neighbour tensor force model with measured phonon frequencies. There are five peaks at frequencies of about 4.58 , $5.60,5.77,5.99$ and 7.61 (all in THz). The maximum frequency is $8.00 \mathrm{THz}$. Comparing our results (Fig. 3), we find the positions of the respective peaks and overall shape closely resembling the experimental findings [44]. However, it should be noted that our fourth peak is higher than that observed in the experiment.

The experimental determination of $C_{\mathrm{v}}$ in the case of molybdenum has been made by Clusius and Franzosini [34]. There is good agreement between the theoretical and experimental values of electronic specific heat for this metal. Moren and Maita [45] have found $\gamma=4.8 \times 10^{-4} \mathrm{cal} / \mathrm{mol} \mathrm{deg}^{2}$ for the superconducting phase and have shown it to be consistent with the band structure of this metal. The other experimental values for $\gamma$ are $5.1 \pm 0.4$ [37], $5.25 \pm 0.26$ [38] and 5.05 [39], all in $10^{-4} \mathrm{cal} /$ mol deg ${ }^{2}$. In the present study, the lattice specific 


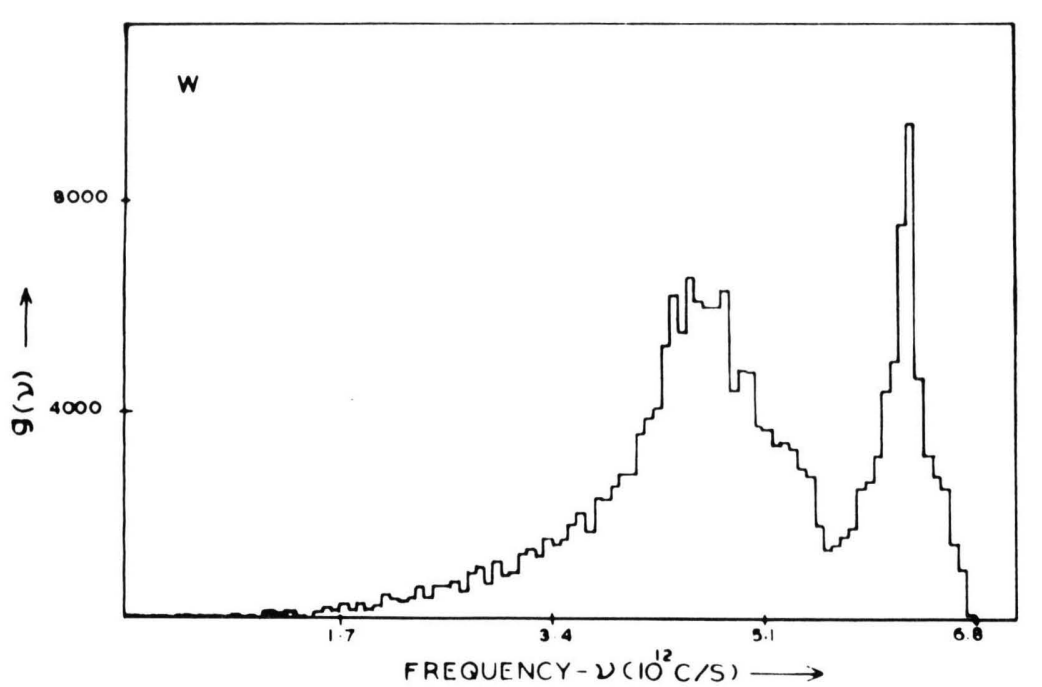

Fig. 1. Phonon density of states in tungsten.

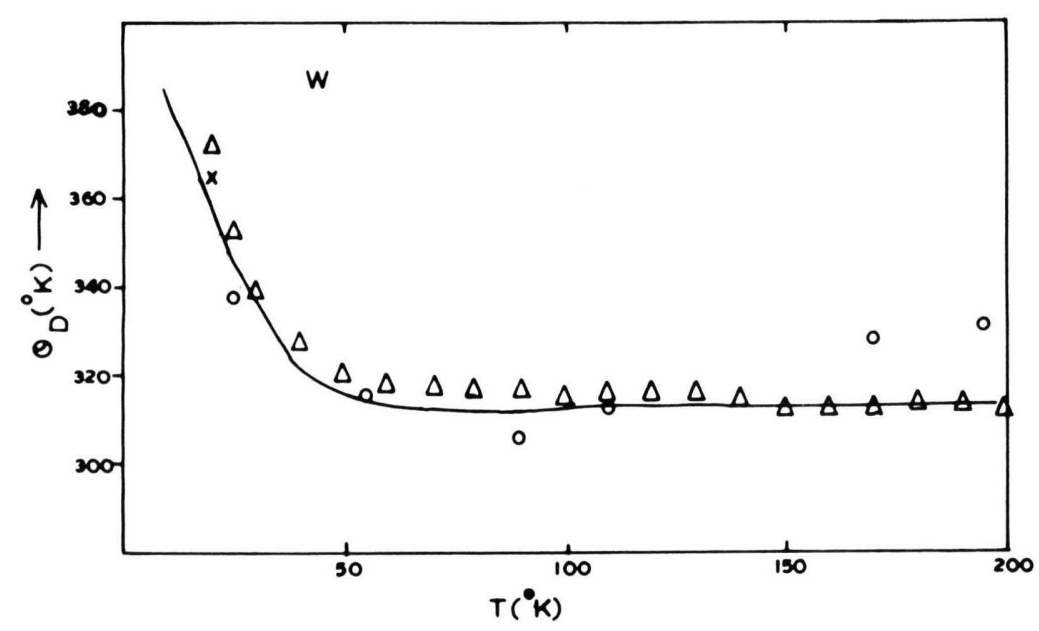

Fig. 2. Debye temperature (solid curve) of tungsten. Experimental points are $\circ[35,36], \triangle[34]$ and $\times[39]$.

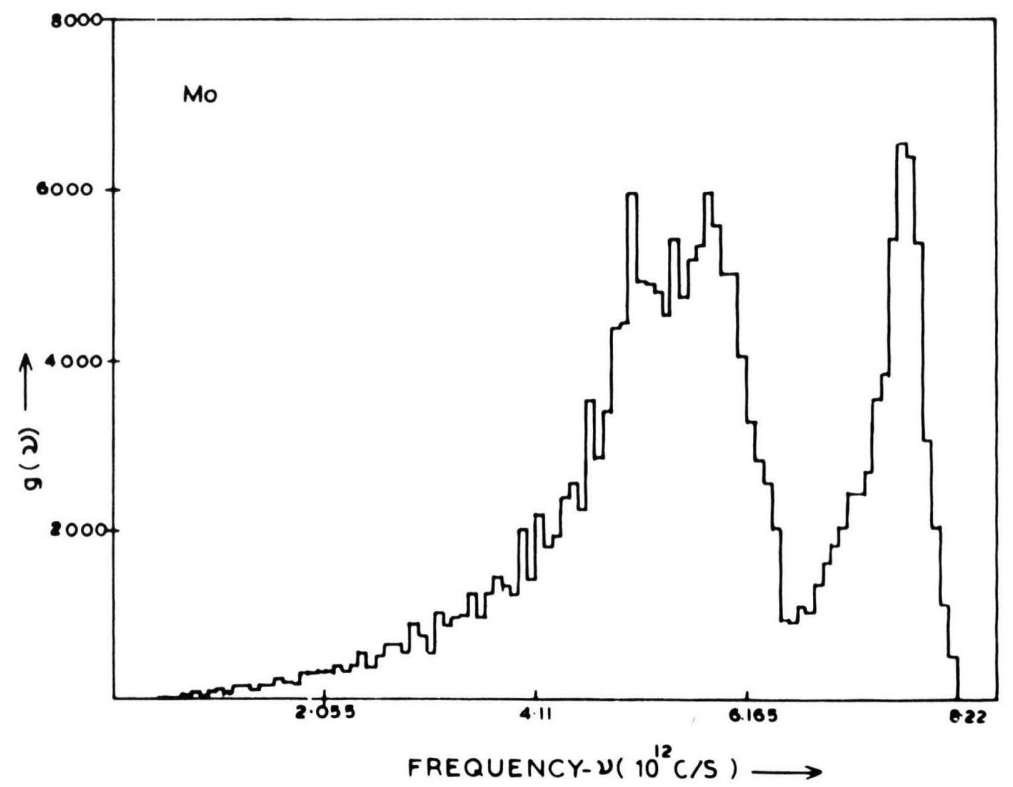

Fig. 3. Frequency distribution function of molybdenum.

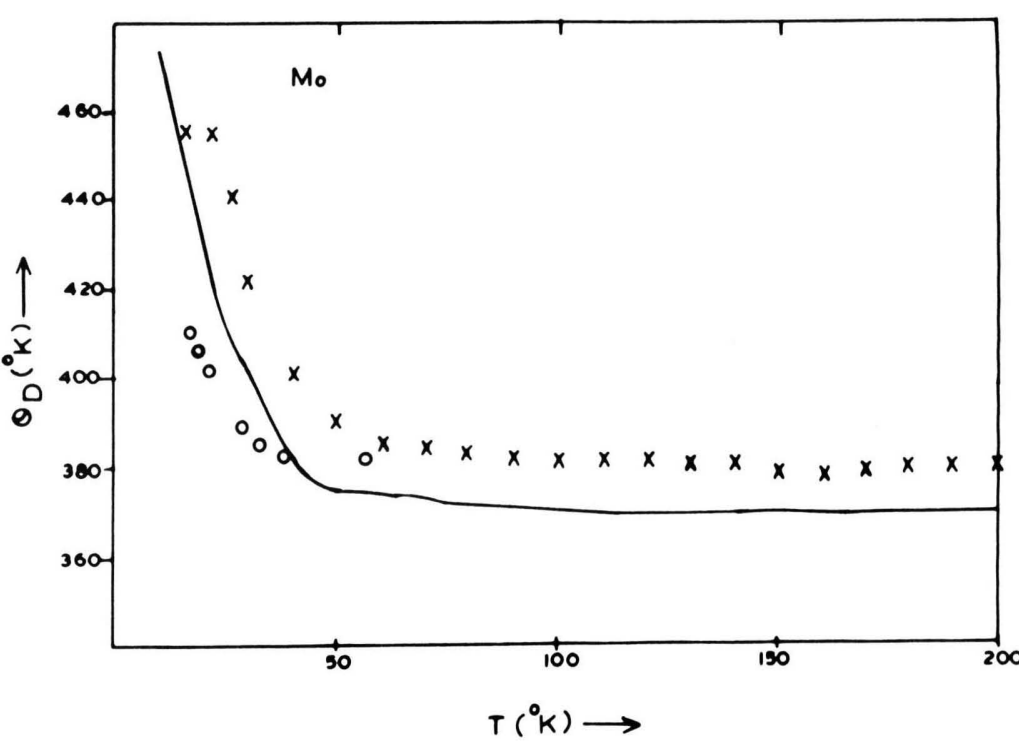

Fig. 4. The Debye temperature vs. temperature curve for molybdenum. Experimental points are $\times[34]$ and $\circ[46]$. 

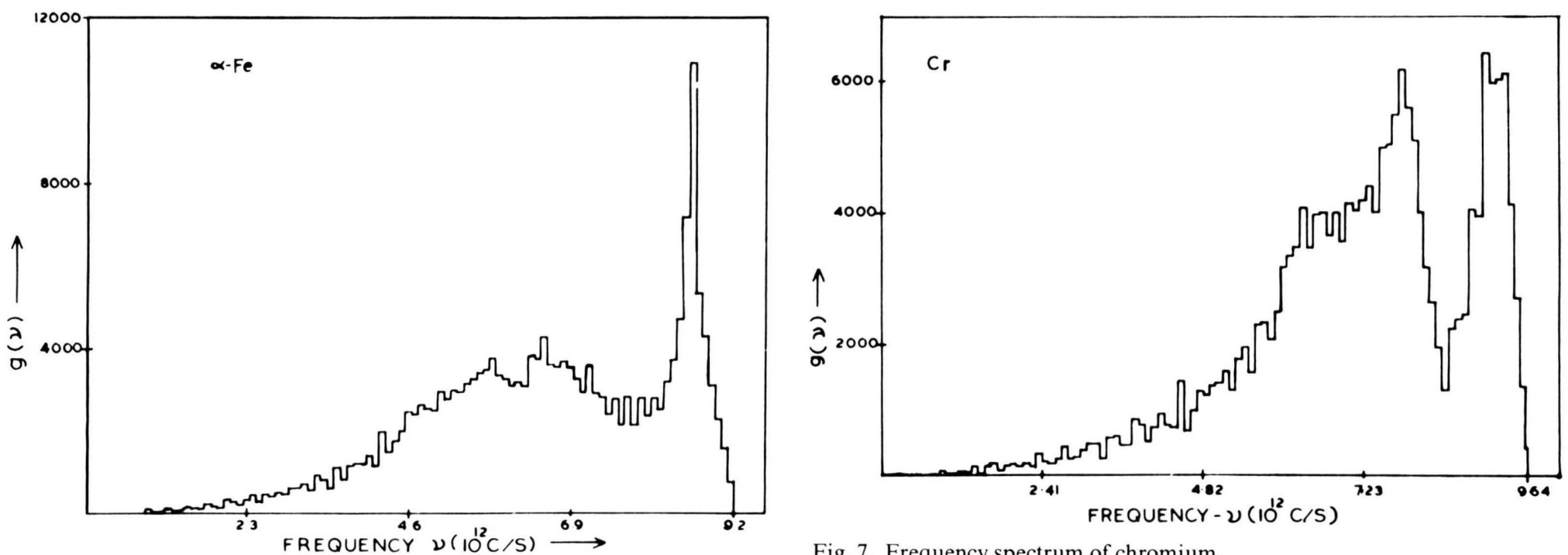

Fig. 5. Density of phonon states for alpha-iron.

Fig. 7. Frequency spectrum of chromium.

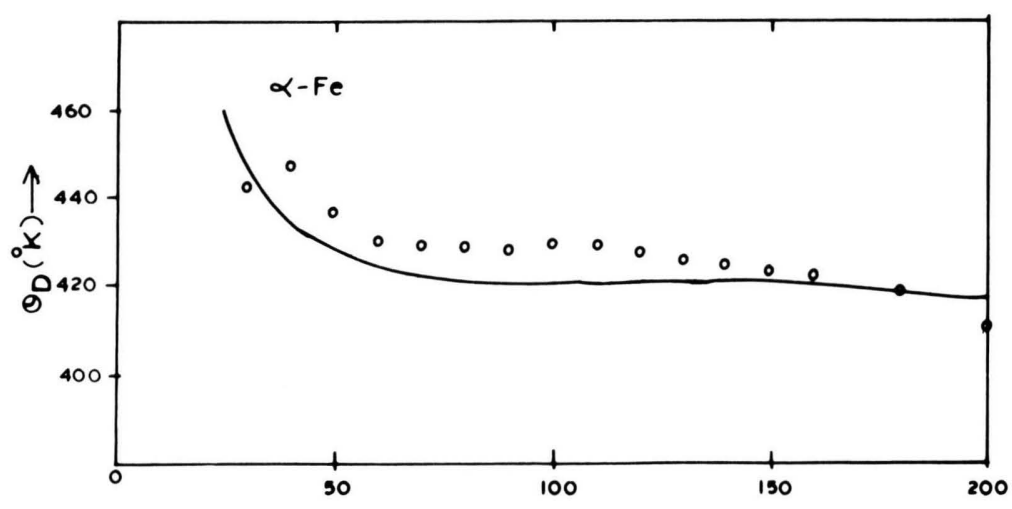

Fig. 6. $\theta_{\mathrm{D}}$ representation of the lattice specific heat for alpha-iron (solid curve). Experimental points ( 0 ) are due to Duyckaerts [50] and Kelley [51].

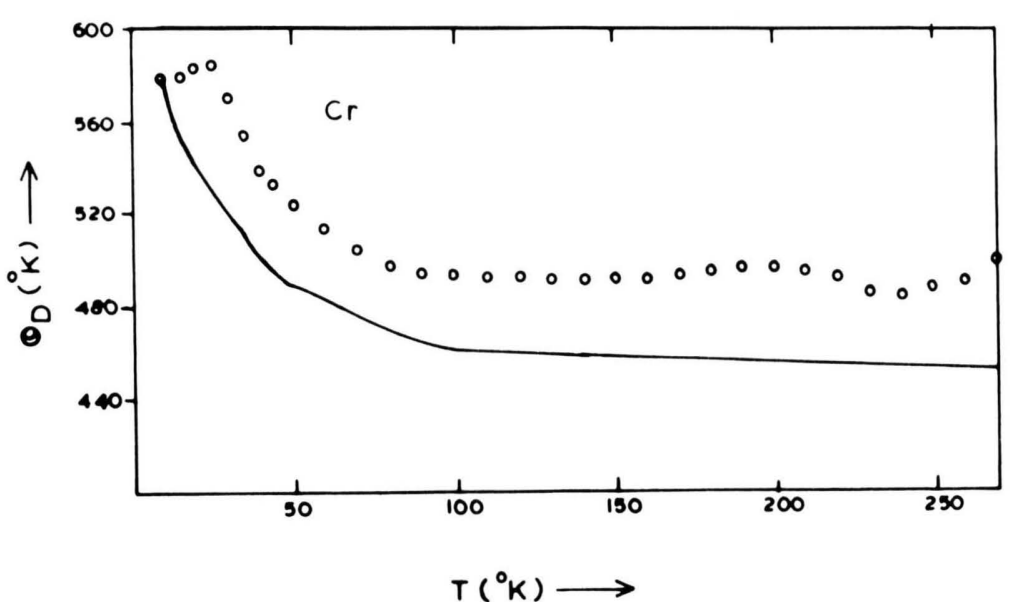

Fig. 8. Debye temperatures of chromium. Experimental points are from Clusius and Franzosini [65] 
heat of molybdenum is calculated by employing the data suggested by Clusius and Franzosini [34].

The comparison of experimental data made in terms of the $\theta_{\mathrm{D}}$ is shown in Figure 4 . The $\theta_{\mathrm{D}}$ values from the earlier work [46] have also been shown. It seems that the theoretical values from the present work agree fairly well with the experimental findings and are found to be better than those reported by Behari and Tripathi [24].

Alpha-iron: There have been several attempts [6, $47,48]$ to obtain the frequency spectrum of this metal from general force constant models determined from inelastic neutron scattering data. Minkiewicz et al. [6] have employed a fifth neighbour general force constant model to analyze the neutron inelastic scattering data. They have used the method of Gilat and Raubenheimer [49] to calculate the frequency spectrum. We preferred the above experimental results [6] for the comparison, as all the measurements are almost in agreement with each other. There are three peaks at frequencies about 5.66, 6.55 and 8.70 (all in THz). Our results (Fig. 5) agree very well with the experimental report. As regards the maximum frequency at about $9.3 \mathrm{THz}$ and the relative heights of different peaks, the present results are found to be in good agreement with the experimental values.

The experimental values of $C_{\mathrm{v}}$ have been taken for temperatures below and above $50 \mathrm{~K}$, respectively $[50,51]$. The observations of Kelley [51] have been adopted as these are in broad agreement with other reports [52, 53]. Manning [54], from band structure studies, has computed the value of $\gamma$ to be

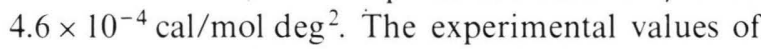
$\gamma 12.06[50], 12 \pm 0.12$ [55] and $11.91 \pm 0.22$ [56], all in $10^{-4} \mathrm{cal} / \mathrm{mol} \mathrm{deg}^{2}$, are definitely much larger than the theoretical one. Rayne and Chandrashekhar [57] have re-analysed the data of Cheng et al. [56] assuming the presence of a spin wave contribution to the heat capacity at low temperatures. They have used their low temperature elastic data extrapolated to zero Kelvin to evaluate the lattice contribution to the heat capacity, which is subtracted from the experimental value. The analysis of the residual heat capacity yields the values $(11.7 \pm 0.1) \times 10^{-4} \mathrm{cal} /$ $\mathrm{mol} \mathrm{deg}{ }^{2}$ and $(2 \pm 1) \times 10^{-5} \mathrm{cal} / \mathrm{mol} \mathrm{deg}{ }^{5 / 2}$ from the coefficients of electronic and spin wave contributions, respectively. The presence of the spin wave contributions to the heat capacity for this metal have also been confirmed experimentally [58, 59]. Dixon et al: [60] have calculated this contribution from the exchange interaction term derived from neutron scattering data and have subtracted it from their calorimetric value. We have employed the results due to Dixon et al. [60] to evaluate the electronic and lattice wave contributions, which have been subtracted from the experimental data to give the lattice specific heats.

The $\theta_{\mathrm{D}}$ vs. $T$ curve obtained in the present study (Fig. 6) exhibits good agreement with the observed data. Here also, the results are found to be better than the most recent theoretical findings [61].

Chromium: A tensor force model, including interactions up to four nearest neighbours, fitted with inelastic neutron diffraction data and elastic constant data, has been employed by Feldman [62] to calculate the frequency spectrum of chromium. Shaw and Muhlestein [4] have also shown a histogram representation of the frequency distribution function using fourth nearest neighbour Born-von Karman analysis fitted to experimental phonon frequencies. Figure 7 displays the theoretical frequency spectrum due to the present work. There are two peaks in the experimental spectrum [4] at frequencies of about 7.88 and $9.25 \mathrm{THz}$. The positions of the peaks and maximum experimental frequency $(9.6 \mathrm{THz})$ are in close agreement with present results (Figure 7). Still the present spectrum differs from the experiment in one respect. In the theoretical curve the first peak is not higher than the second one. This is contrary to the experimental observations $[4,62]$. Still, however, it should be noted that both peaks in the results have nearly the same height. The calculated spectrum is better than that reported by Pal [63] on the basis of Sharma-Joshi model [64].

Clusius and Franzosini [65] have experimentally determined the specific heats of chromium. The values of $\gamma$ used in the present calculation are $360 \times 10^{-6} \mathrm{cal} / \mathrm{mol} \mathrm{deg}^{2}$ up to $20 \mathrm{~K}$ and $138 \times 10^{-6}$ $\mathrm{cal} / \mathrm{mol} \mathrm{deg}{ }^{2}$ above $60 \mathrm{~K}$. The corresponding values of $\theta_{\mathrm{D}}$ have been plotted (Fig. 8) after correction for the electronic specific heat contributions. It can be seen from Fig. 8 that the experimental and theoretical $\theta_{\mathrm{D}}$ vs. $T$ curves show a similar trend. Other theoretical studies did also yield the same type of trend $[63,66]$. 
Tantalum: Wood [3] has computed a frequency spectrum for this metal using a seventh neighbour general force model fitted to the phonon frequencies measured by neutron inelastic scattering. There are two peaks at frequencies of about 2.77 and $4.33 \mathrm{THz}$. Comparing the present results (Fig. 9) we find the positions of the respective peaks and overall shape closely resembling the results of Woods [3]. The relative heights of the peaks are also in agreement. The results are also comparable with those reported by Pathak et al. [27]. These authors have calculated the frequency spectrum employing a five force constant model and mesh size $20 \times 20 \times 20$.

Specific heat data for tantalum have been taken from the work of Clusius and Losa [68]. The $\gamma$ value, used for the present calculation is $760 \times 10^{-6} \mathrm{cal} /$ $\mathrm{mol} \mathrm{deg}^{2}$. It can be seen from Fig. 10 that the theoretical and experimental $\theta_{\mathrm{D}}$ vs. $T$ curves are in broad agreement. A comparison with previously reported theoretical data shows that the present results are as good as those of Gupta et al. [67] and are better than those reported by Sharma et al. [66].

Vanadium: The analysis of the phonon dispersion relations with the Born-von Karman force constant model indicates that interatomic forces in vanadium are complicated and are of a fairly long range. At least seven nearest neighbour interactions are needed to fit the data [1]. The incomplete d electronic shell causes many unusual properties [69], particularly a large cohesive energy [70], and might be expected to have a significant effect on the lattice dynamics of vanadium.

The phonon frequency distribution of vanadium has been studied through inelastic neutron scattering techniques by many workers [71-81] but nothing has been done with high precision to locate the positions of the critical points. Early workers have obtained only one peak because of their poor instrumental resolution. Probably the most accurate experimental determination of the frequency spectrum has been reported by Page [81]. Collela and Batterman [1] have also reported the frequency distribution of vanadium. The computations were made in two ways: (i) The force constants were determined by a least squares fit with all the frequencies. (ii) The force constants were determined from the interplanar force constants. The latter frequency spectrum (ii) is believed to be less accurate as it may reflect the experimental uncertainty in determining the dispersion curves [1].
It is interesting to compare the present theoretical results (Fig.11) with those reported by Page [81] and Collela and Batterman [1]. The frequency spectrum due to Page [81] exhibits four peaks at frequencies $1.9,4.9,6.1$ and 6.9 (all in $\mathrm{THz}$ ). A peak in the distribution at a frequency of about $2 \mathrm{THz}$ has been predicted by Pelah et al. [82] and attributed to the Kohn effect. Moreover, the frequency spectrum obtained by Page [81] shows a tail at higher frequencies.

The X-ray frequency spectrum of Collela and Batterman [1] does not show any hump in the low frequency region. The low frequency region is experimentally the most accurate, since the thermal scattering is high here, and the extra contributions are a small fraction of the total measured intensity. There are four peaks in the frequency spectrum at frequencies of about 4.42, 5.58, 6.44 and 6.97 (all in $\mathrm{THz}$ ). The first peak is the highest one. It can be seen (Fig. 11) from the present theoretical frequency spectrum that the positions of the peaks are at 4.94, 5.37, 5.63 and 6.24 (all in THz). There is one more peak at $6.58 \mathrm{THz}$ in the calculated spectrum. Therefore the present theoretical curve differs slightly from the experimental reports [1,81] with respect to the locations of the peaks. it is interesting to watch our theoretical spectrum in the sense that it extends in high frequency region. No doubt that this tail is not at all as pronounced as was observed by Page [81]. It is believed that this high energy tail observed in the experiment might be due to improper correction for multiphonon processes $[1,86]$. However, the present frequency distribution for vanadium agrees fairly well with the experimental findings $[1,82]$, especially in the low frequency region. Many attempts [23, 30, 63, 83-87] have been made to calculate the frequency spectrum of vanadium. Singh and Bowers [84] have employed a non-central three force constant model using the hypothetical elastic constants deduced from the low temperature specific heat data. The calculation of Allers [85], based on the central two force constant model shows disagreement with experiment. Sharma [88] has calculated the frequency spectrum of vanadium on the basis of the de Launay electron gas model [89] using the experimental values of the elastic constants. His spectrum also does not agree with the experiments. The spectra of Hendricks et al. [86] using a noncentral four force constant model also does not agree well with the experiments. The 

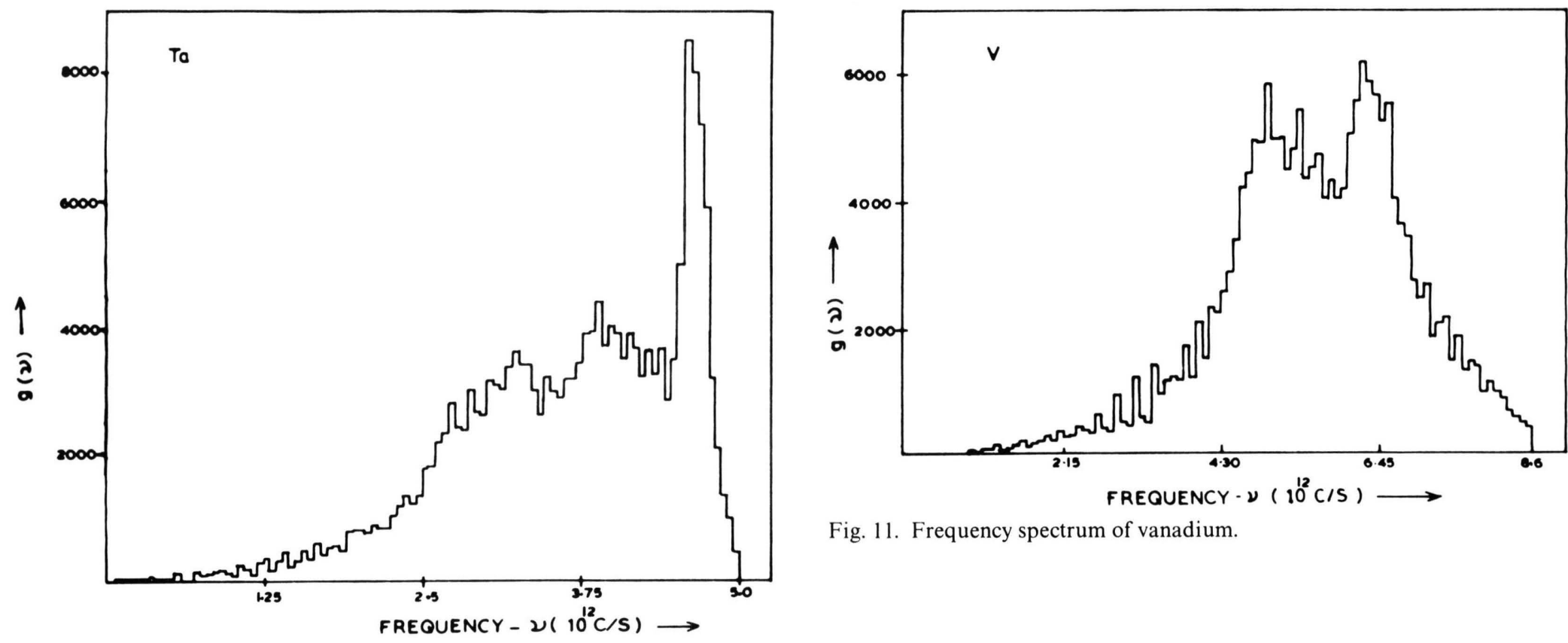

Fig. 11. Frequency spectrum of vanadium.

Fig. 9. Phonon density of states in tantalum.

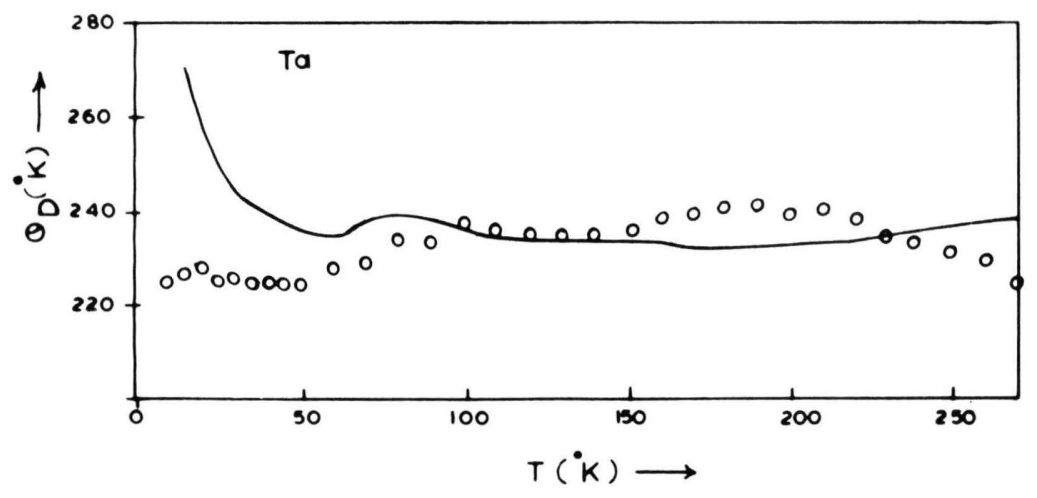

Fig. 10. Debye temperatures of tantalum. Experimental points are due to Clusius and Losa [68].

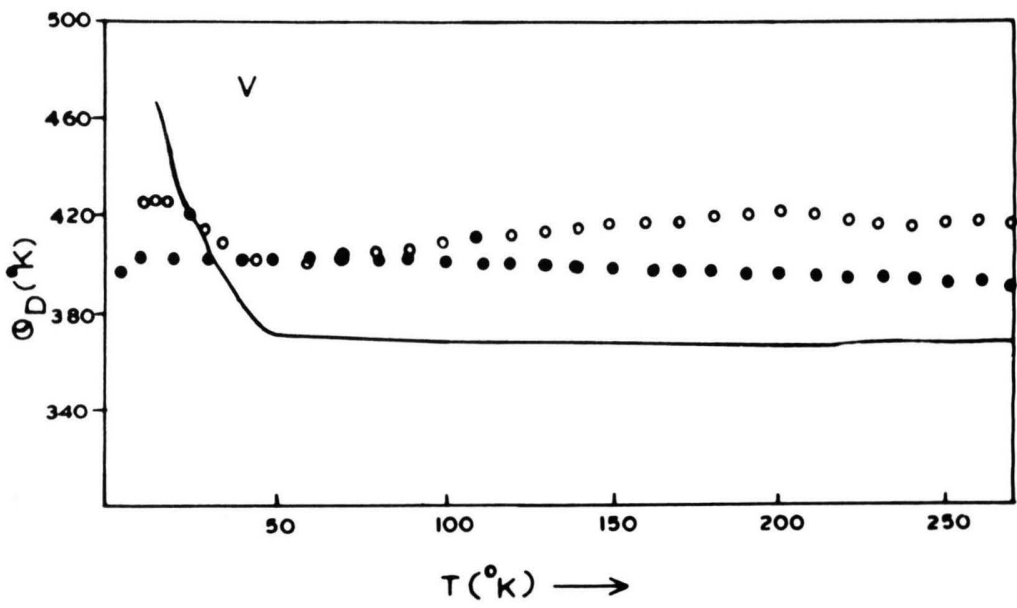

Fig. 12. Debye temperatures of vanadium. Experimental results are from: (a) $\circ$ Clusius et al. [90] and (b) $\bullet$ Bolef et al. [91]. 
calculations of Pal [63] using the Sharma-Joshi [64] model show fair agreement with the experiments. The frequency spectra obtained by Kharoo et al. [30] and Gupta and Hemkar [63] employing a five force constant model show comparatively better agreement with the experiments than the above theoretical findings [88, 30, 63, 84-87].

The experimental data of the lattice specific heat for this metal are taken from the work of Clusius et al. [90]. Experimental points have been considered after subtracting the electron heat contribution. The coefficient $\gamma$ for this correction is 1600 below $20 \mathrm{~K}$ and 1500 above $100 \mathrm{~K}$ in units of $10^{-6} \mathrm{cal} / \mathrm{mol} \mathrm{\textrm {deg } ^ { 2 }}$ due to Clusius et al. [90]. The corresponding $\theta_{\mathrm{D}}$ along with the theoretical $\theta_{\mathrm{D}}$ of the present study are plotted in Figure 12. The latest Debye temperature $\theta_{\mathrm{D}}$ data reported by Bolef et al. [91] have also been shown (Figure 12). There is a good qualitative agreement between the theoretical and experimental curves. The marked discrepancy between theory and experiments, particularly in the case of vanadium may be associated with the uncertainties involved in the electron and spin wave contributions to the lattice specific heats.

[1] R. Collela and B. W. Batterman, Phys. Rev. B 1, 3913 (1980).

[2] R. I. Sharp, J. Phys. C 2, 421 (1969).

[3] A. D. B. Woods, Phys. Rev. 136, A 781 (1964).

[4] W. M. Shaw and L. D. Muhlestein, Phys. Rev. B 4, 969 (1971).

[5] S. H. Chen and B. N. Brockhouse, Solid State Commun. 2, 73 (1964).

[6] V. J. Minkiewicz, G. Shirane, and R. Nathans, Phys. Rev. 162, 528 (1967).

[7] A. D. B. Woods and S. H. Chen, Solid State Commun. 2, 233 (1964).

[8] A. P. Miller and B. N. Brockhouse, Can. J. Phys. 49, 704 (1971).

[9] B. M. Powell, P. Martel, and A. D. B. Woods, Phys. Rev. 171, 727 (1968).

[10] F. J. Disslo and J. A. Wilson, Adv. Phys. 24, 117 (1975).

[11] A. Larose and B. N. Brockhouse, Can. J. Phys. 54, 1819 (1976).

[12] R. J. Birgeneau, J. Cordes, G. Dolling, and A. D. B. Woods, Phys. Rev. 136, A 1359 (1964).

[13] D. H. Dutton, B. N. Brockhouse, and A. P. Miller, Can. J. Phys. 50, 2915 (1972).

[14] J. Zarestky, C. Stassis, B. N. Harmon, K. M. Ho, C. L. Fu, Phys. Rev. B 28, 697 (1983).

[15] V. B. Gohel and A. R. Jani, Physica 82 B, 33 (1976).

[16] V. B. Gohel and A. R. Jani, Pramana. 9, 593 (1977).

[17] V. B. Gohel and A. R. Jani, Ind. J. Phys. 50 A, 609 (1976).

[18] V. B. Gohel and A. R. Jani, Aust. J. Phys. 29, 31 (1976).
A critical examination of the present theoretical results reveals that the phonon density of states and temperature variations of $\theta_{\mathrm{D}}$ obtained from the phenomenological theory [16] provide a quite satisfactory description of the experimental information. However, the apparent small discrepancy between theory and experiment is not unexpected and can be attributed to the temperature variation of the elastic constants and the lattice parameter and the neglect of anharmonic effects. Still, it may be emphasized that the improvement of the present results over previously reported theoretical work strongly justifies the use of finer mesh $(40 \times 40 \times 40)$ to evaluate the phonon density of states by a good phenomenological model.

\section{Acknowledgements}

The author wishes to thank Dr. V. B. Gohel (Gujarat University) for his valuable help in this work. Thanks are also due to Professor M. K. Agarwal (Head of the Department) for his support and encourangement to carry out this work. Financial assistance from UGC, New Delhi is gratefully acknowledged here.

[19] M. Blackman, Proc. Roy. Soc. A 159, 416 (1937).

[20] M. Blackman, Encyclopaddeia of Physics, Bd. 7, Springer Verlag, Berlin 1955, p. 325.

[21] A. R. Jani, Ph.D. Thesis, Gujarat University 1979, p. 76.

[22] R. Cavalheiro and M. M. Shukla, Acta Phys. Pol. A A 49, 27 (1976).

[23] P. K. Sharma, S. Pal, and R. P. Gupta, Rev. Roumaine Phys. 14, 247 (1969).

[24] J. Behari and B. B. Tripathi, J. Phys. Soc. Japan 33, 1207 (1972).

[25] J. Prakash, L. P. Pathak, and M. P. Hemkar, Aust. J. Phys. 28, 63 (1975).

[26] L. P. Pathak, V. P. Singh, J. Prakash, and M. P. Hemkar, Ind. J. Phys. 51 a, 42 (1977).

[27] L. P. Pathak, R. C. Rai, and M. P. Hemkar, J. Phys. Soc. Japan 44, 1834 (1978).

[28] P. S. Mahesh and B. Dayal, Phys. Rev. 143, 443 (1966)

[29] A. K. Singh and P. K. Sharma, J. Phys. Soc. Japan 26, 425 (1969).

[30] H. L. Kharoo, O. P. Gupta, and M. P. Hemkar, Chem. Phys. Lett. 52, 509 (1977).

[31] H. L. Kharoo, O. P. Gupta, and M. P. Hemkar, Czech. J. Phys. B 28, 77 (1978).

[32] N. Singh and P. K. Sharma, Phys. Rev. B 3, 1141 (1971).

[33] A. K. Ghatak and L. S. Kothari, An Introduction to Lattice Dynamics. Addison-Wesley Publ. Co., 1972, p. 140.

[34] K. Clusius and P. Franzosini, Z. Naturforsch. 14a, 99 (1959). 
[35] F. Lange, Z. Physik. Chem. 110, 343 (1924).

[36] C. Zwikker, Z. Physik 52, 668 (1928).

[37] M. Horowitz and J. G. Daunt, Phys. Rev. 91, 1090 (1953).

[38] F. Rayne, Phys. Rev. 95, 1428 (1954).

[39] N. M. Walcot, Inconference de physique des Basses Temperatures, Centre National de $\mathrm{La}$ Recherche Scientifique and UNESCO, Paris, P 206 (1956).

[40] T. R. Waite, R. S. Craig, and W. E. Wallace, Phys. Rev. 104, 5 (1956).

[41] T. R. Waite, R. S. Craig, and W. E. Wallace, Phys. Rev. 104, 1240 (1956).

[42] M. F. Manning and M. I. Chodorow, Phys. Rev. 56, 787 (1939).

[43] C. B. Walker and P. A. Egelstaff, Phys. Rev. 177, 1111 (1969).

[44] B. M. Powell, P. Mortel, and A. D. B. Woods, Can. J. Phys. 55, 1601 (1977).

[45] F. J. Morin and J. P. Maita, Phys. Rev. 129, 1115 (1963).

[46] F. Simon and W. Zeidler, Z. Physik. Chem. 123, 383 (1926).

[47] B. N. Brockhouse, H. E. Abou-Helal, and E. D. Hallmen, Sol. State Commun. 5,211 (1967).

[48] J. Bergsma, C. Van Dijak, and D. Jocchetti, Phys. Lett. 24 A, 270 (1967).

[49] G. Gilat and L. J. Raubenheimer, Phys. Rev. 144, 390 (1966).

[50] G. Duyckaerts, Physica 6, 401 (1939).

[51] K. K. Kelley, J. Chem. Phys. 11, 16 (1943).

[52] A. Eucken and H. Werth, Z. Anorg. Allgem. Chem. 188, $152(1930)$

[53] F. Simon and R. C. Swain, Z. Physik. Chem. B 28, 189 (1935)

[54] M. F. Manning, Phys. Rev. 63, 190 (1943).

[55] W. H. Keesom and B. Kurrelmeyer, Physica 6, 663 (1939).

[56] C. H. Cheng, C. T. Wei, and P. A. Beck, Phys. Rev. 120, 426 (1960).

[57] J. A. Rayne and B. S. Chandrashekhar, Phys. Rev. 122, $1715(1961)$

[58] P. E. Tennenwald, Proceedings of the International Conference on Magnetism and Crystallography, The Physical Society of Japan, Kunkyo-Ku, Tokyo 1962, p. 592.

[59] H. Heatherly, K. Jirikawa, R. D. Lowde, J. F. Mallet, M. W. Stringfellow, and B. H. Torrie, J. Appl. Phys. 35, 802 (1964).

[60] M. Dixon, F. E. Hoare, T. M. Holden, and D. E. Moody, Proc. Roy. Soc. London A 285, 561 (1965).

[61] O. P. Gupta, Acta Phys. Pol. A A 64, 269 (1983).

[62] J. L. Feldman, Phys. Rev. 1, 448 (1970).

[63] S. Pal, Phys. Rev. B 9, 5144 (1974).

[64] P. K. Sharma and S. K. Joshi, J. Chem. Phys. 39, 2633 (1963).
[65] K. Clusius and P. Franzosini, Z. Naturforsch. 17a, 522 (1962).

[66] P. K. Sharma, B. S. Semwal, and K. N. Mehrotra, Z. Naturforsch. 26a, 747 (1971).

[67] O. P. Gupta, H. L. Kharoo, and M. P. Hemkar, Pramana 11, 111 (1978).

[68] K. Clusius and G. Losa, Z. Naturforsch. 10a, 939 (1955).

[69] N. F. Mott, Advan. Phys. 13, 325 (1964).

[70] N. F. Mott, Rep. Prog. Phys. 25, 218 (1962).

[71] R. S. Carter, D. J. Hughes, and H. Palevsky, Phys. Rev. 104, 271 (1956).

[72] B. N. Brockhouse, Can. J. Phys. 33, 889 (1955).

[73] A. T. Stewart and B. N. Brockhouse, Rev. Mod. Phys. 30, 250 (1958).

[74] C. M. Eisenhauer, I. Pelah, J. Hughes, and H. Palevsky, Phys. Rev. 109, 1046 (1958).

[75] K. C. Turberfield and P. A. Egelstaff, Phys. Rev. 127, 1017 (1962)

[76] R. Haas, W. Kley, K. H. Krebs, and R. Rubin, Proceedings of the Symposium on Inelastic Scatterings of Neutron in Solid States and Liquids, Vienna 2, 145 (1963).

[77] M. G. Zemlyanov, Y. M. Kagan, N. A. Techernoplekov, and A. G. Tchetserin, Proceedings of the Symposium on Inelastic Scattering of Neutrons in Solid States and Liquids, Vienna 2, 145 (1963).

[78] W. Glaser, F. Carvolho, and G. Eheet, Proceedings of the Symposium on Inelastic Scattering of Neutrons in Solid State and Liquid, Vienna 1,99 (1965).

[79] B. Mozer, K. Otnes, and H. Palevsky, J. Phys. Chem Sol. Suppl.1, 63 (1965).

[80] G. Dolling and A. D. B. Woods, Thermal Neutron Scattering, ed. by P. A. Eglestaff, New York 1965, p. 210 .

[81] D. J. Page, Proc. Phys. Soc. London 91, 76 (1967).

[82] I. Pelah, R. Hass, W. Kley, K. H. Krebs, J. Peretti, and R. Rubin, Inelastic Scattering of Neutrons in Solids and Liquids, Vienna 2, 155 (1963).

[83] O. P. Gupta and M. P. Hemkar, Nuovo Cimento B 45 B, 255 (1978).

[84] D. N. Singh and W. A. Bowers, Phys. Rev. 116, 116 (1959).

[85] G. A. Allers, Phys. Rev. 119, 1532 (1960).

[86] J. B. Hendricks, H. N. Riser, and C. B. Clark, Phys. Rev. 130, 1377 (1963).

[87] B. C. Clark, D. C. Gazis, and R. F. Wallis, Phys. Rev. 134, A 1486 (1964).

[88] B. Sharan, J. Chem. Phys. 36, 1116 (1962).

[89] J. de Launay, Solid State Phys. (AP) 2, 219 (1956).

[90] K. Clusius, P. Franzosini, and U. Piesbergen, Z. Naturforsch. 15a, 728 (1960).

[91] D. I. Bolef, R. E. Smith, and J. G. Miller, Phys. Rev. B 3, 4100 (1971). 